\title{
gु \\ Spin gaps and spin-flip energies in density-functional theory
}

\author{
K. Capelle, ${ }^{1,2}$ G. Vignale, ${ }^{3}$ and C. A. Ullrich ${ }^{3}$ \\ ${ }^{1}$ Centro de Ciências Naturais e Humanas, Universidade Federal do ABC (UFABC), Santo André 09210-170, SP, Brazil \\ ${ }^{2}$ Instituto de Física de São Carlos, Universidade de São Paulo, São Carlos 13560-970, SP, Brazil \\ ${ }^{3}$ Department of Physics and Astronomy, University of Missouri-Columbia, Columbia, Missouri 65211, USA \\ (Received 9 November 2009; revised manuscript received 18 February 2010; published 16 March 2010)
}

\begin{abstract}
Energy gaps are crucial aspects of the electronic structure of finite and extended systems. Whereas much is known about how to define and calculate charge gaps in density-functional theory (DFT), and about the relation between these gaps and derivative discontinuities of the exchange-correlation functional, much less is known about spin gaps. In this paper we give density-functional definitions of spin-conserving gaps, spin-flip gaps and the spin stiffness in terms of many-body energies and in terms of single-particle (Kohn-Sham) energies. Our definitions are as analogous as possible to those commonly made in the charge case, but important differences between spin and charge gaps emerge already on the single-particle level because unlike the fundamental charge gap spin gaps involve excited-state energies. Kohn-Sham and many-body spin gaps are predicted to differ, and the difference is related to derivative discontinuities that are similar to, but distinct from, those usually considered in the case of charge gaps. Both ensemble DFT and time-dependent DFT (TDDFT) can be used to calculate these spin discontinuities from a suitable functional. We illustrate our findings by evaluating our definitions for the Lithium atom, for which we calculate spin gaps and spin discontinuities by making use of near-exact Kohn-Sham eigenvalues and, independently, from the single-pole approximation to TDDFT. The many-body corrections to the Kohn-Sham spin gaps are found to be negative, i.e., single-particle calculations tend to overestimate spin gaps while they underestimate charge gaps.
\end{abstract}

DOI: 10.1103/PhysRevB.81.125114

PACS number(s): 71.15.Mb, 31.15.ej, 31.15.ee, 72.25.Dc

\section{INTRODUCTION}

There is hardly any electronic property of a system that does not depend on whether there is an energy gap for charge excitations, or for particle addition and removal. Similarly, there is hardly any magnetic property of a system that does not depend in some way on whether there is an energy gap for flipping a spin, or for adding and removing spins from the system.

The reliable calculation of charge gaps ${ }^{1}$ from first principles is nontrivial and still faces practical problems (relevant aspects are reviewed below), but at least conceptually it is clear how charge gaps are to be defined and quantified within modern electronic-structure methods, such as densityfunctional theory (DFT) ${ }^{2-4}$ On the other hand, much less is known about how to calculate, or even define, spin gaps.

In the present paper we show how to define and calculate the spin gap in spin-DFT (SDFT), and predict that such calculations will encounter a spin-gap problem similar to the band-gap problem familiar from applications of DFT to semiconductors or to strongly correlated systems.

Section II of this paper is devoted to charge gaps. In Sec. II A we recapitulate the conceptual difference between fundamental gaps and excitation gaps. In Sec. II B we then recall the quantitative definition of the fundamental gap and related quantities, such as the single-particle gap, and particle addition and removal energies. Section II C summarizes key aspects of the derivative discontinuity, while Sec. II D describes the connection between gaps and discontinuities within the framework of ensemble DFT. Although the final results of these sections are well known, our treatment is different from the usual one in so far as we introduce manybody corrections to the gap and derivative discontinuities in completely independent ways, related only a posteriori via ensemble DFT. This way of proceeding is useful for performing the generalization to the spin case.

For both the fundamental gap and the optical excitation gap, the gapped degree of freedom is related to particles: either particles are added to or removed from the system, or particles are excited to higher energy levels within the system under study. In ordinary atoms, molecules, and solids, these particles are electrons, and the particle gaps of manyelectron systems are a key property in determining the functionality of today's electronic devices.

The last decade has witnessed an enormous growth of interest in another type of system, and in devices resulting from them, in which the key degree of freedom is the spin. In the resulting field of spintronics, and the development of spintronic devices, one is interested in controlling and manipulating the spin degrees of freedom independently of, or in addition to, the charge degrees of freedom. Here, the issue of the spin gap arises, and a number of questions for electronic-structure and many-body theory appear: what is the energy required to add a spin to the system? What is the energy cost of flipping a spin? How do these concepts differ from the fundamental and optical gaps involving particles? Can we calculate spin gaps from spin-density-functional theory, and if yes, what type of exchange-correlation $(x c)$ functional is required? In Sec. III, we answer these questions.

In Sec. III A we contrast spin gaps with charge gaps, and in Sec. III B we propose a set of many-body and singleparticle definitions for quantities related to the spin gap, such as spin-flip energies and the spin stiffness. We take care to ensure that all quantities appearing in our definitions can, in principle, be calculated from conventional SDFT or timedependent SDFT (TDSDFT), and try to make the definitions 
in the spin case as analogous as possible to the charge case. However, this analogy can only be carried up to a certain point, and important differences between charge gaps and spin gaps emerge already at this level. As a simple example, we consider, in Sec. III C, the Lithium atom, for which we confront calculated and experimental spin gaps.

In Sec. III D we then use ensemble DFT to relate the spin gap to a derivative discontinuity that is similar to, but distinct from, the one usually considered in the charge case. Finally, in Sec. III F, we investigate the connection to excitation gaps calculated from TDSDFT. Equations are given that allow one to extract the various spin gaps and related quantities from noncollinear TDSDFT calculations. For illustrative purposes we evaluate these for the Lithium atom, and compare the gaps and discontinuities obtained from time-dependent DFT to those obtained in Sec. III C from time-independent considerations. Section IV contains our conclusions.

\section{CHARGE GAP}

\section{A. Fundamental gaps vs excitation gaps}

To provide the background for this investigation, let us first briefly recapitulate pertinent aspects of charge (or particle $^{1}$ ) gaps. While by definition all gaps involve energy differences between a lower-lying state (in practice often the ground state) and a state of higher energy, important differences depend on how the extra energy is added to the system and what degrees of freedom absorb it. Therefore, different notions of gap are appropriate for different purposes. For processes in which particles are added to or removed from the system, which is subsequently allowed to relax to the ground state appropriate to the new particle number, the key quantity is the fundamental gap (sometimes also called the quasiparticle gap) which is calculated from differences of ground-state energies of systems with different particle number. As such, it is relevant for instance in transport phenomena and electron-transfer reactions. If energy is added by means of radiation, on the other hand, the particle number does not change, and the relevant gap is an excitation energy of the $N$-particle system. This excitation gap (sometimes also called the optical gap), is relevant, e.g., in spectroscopy.

In first-principles electronic-structure calculations, excitation gaps are today often calculated from time-dependent density-functional theory (TDDFT). Fundamental gaps, on the other hand, involve ground-state energies of systems with different particle numbers, and should thus, in principle, be accessible by means of static (ground-state) DFT. However, it is well known that common approximations to DFT encounter difficulties in this regard. In semiconductors, for example, calculated fundamental gaps are often greatly underestimated relative to experiment, and in strongly correlated systems such as transition-metal oxides, gapped materials are frequently incorrectly predicted to be metallic, i.e., to have no gap at all. The resulting band-gap problem of DFT has been intensely studied for many decades.

A major breakthrough in this field was the discovery of the derivative discontinuity of the exact exchange-correlation $(x c)$ functional of DFT, which was shown to account for the difference between the gap obtained from solving the single- particle Kohn-Sham (KS) equations of DFT, and the true fundamental gap. ${ }^{5-7}$ The problems occurring in practice for semiconductors and strongly correlated systems are therefore attributed to the fact that common local and semilocal approximations to the exact $x c$ functional do not have such a discontinuity. The development of DFT-based methods allowing to nonempirically predict the presence and size of gaps in many-electron systems continues to be a key issue of electronic-structure theory and computational materials science.

\section{B. Definition of fundamental charge gaps}

The fundamental charge gap $E_{g}$ is defined as the difference

$$
E_{g}(N)=I(N)-A(N),
$$

where the electron affinity (energy gained by bringing in a particle from infinity) and ionization energy (energy it costs to remove a particle to infinity) are defined in terms of ground-state energies of the $N, N+1$ and $N$-1-particle systems, as

$$
\begin{gathered}
A(N)=E(N)-E(N+1), \\
I(N)=E(N-1)-E(N) .
\end{gathered}
$$

The order of terms in these differences is the conventional choice. The definition of the fundamental gap is in terms of processes involving addition and removal of charge and spin. The change in the respective quantum numbers is \pm 1 in $N$, and $\pm 1 / 2$ in $S$. In chemistry, ${ }^{3}$ the average of $I$ and $A$ is identified with the electronegativity of the $N$-particle system, $[I(N)+A(N)] / 2=\chi(N)$.

The corresponding Kohn-Sham gap is defined analogously as

$$
E_{g, K S}(N)=I_{K S}(N)-A_{K S}(N),
$$

where $I_{K S}(N)=E_{K S}(N-1)-E_{K S}(N) \quad$ and $A_{K S}(N)=E_{K S}(N)$ $-E_{K S}(N+1)$. Since the KS total energy is simply the sum of the KS eigenvalues, $E_{K S}=\sum_{k=1}^{N} \epsilon_{k}$, this reduces to $I_{K S}(N)$ $=-\epsilon_{N}(N)$ and $A_{K S}(N)=-\epsilon_{N+1}(N)$, from which one obtains the usual form

$$
E_{g, K S}(N)=\epsilon_{N+1}(N)-\epsilon_{N}(N),
$$

where $\epsilon_{N}(N)$ and $\epsilon_{N+1}(N)$ are the highest occupied and the lowest unoccupied state of the $N$-particle system, respectively.

The fundamental gap can also be written in terms of KS eigenvalues by means of the ionization-potential theorem (sometimes known as Koopmans' theorem of DFT), which states

$$
\begin{gathered}
I(N)=-\epsilon_{N}(N) \\
A(N)=I(N+1)=-\epsilon_{N+1}(N+1),
\end{gathered}
$$

so that $I(N) \equiv I_{K S}(N)$, and

$$
E_{g}(N)=\epsilon_{N+1}(N+1)-\epsilon_{N}(N) .
$$

Note that in contrast with the KS gap (5) these eigenvalues pertain to different systems. 
The relation between both gaps is established by rewriting the fundamental gap as

$$
E_{g}=E_{g, K S}+\Delta_{x c}
$$

which defines $\Delta_{x c}$ as the $x c$ correction to the single-particle gap. By making use of the previous relations we can cast $\Delta_{x c}$ as $^{8-11}$

$$
\Delta_{x c}=\epsilon_{N+1}(N+1)-\epsilon_{N+1}(N)=A_{K S}(N)-A(N) .
$$

The important thing to notice in these expressions is that, due to protection by Koopmans' theorem, the ionization energy does not contribute to the $x c$ correction $\Delta_{x c}$, so that the correction of the affinity and of the fundamental gap are one and the same quantity. Also, note that all of these definitions can be made without any recourse to ensemble DFT and without any mention of derivative discontinuities.

\section{Nonuniqueness and derivative discontinuities}

The basic Euler equation of DFT is ${ }^{2-4}$

$$
\frac{\delta E[n]}{\delta n(\mathbf{r})}=\mu .
$$

Since $E[n]=F[n]+\int d^{3} r v_{\text {ext }}(\mathbf{r}) n(\mathbf{r}) \quad$ and $\quad E_{K S}[n]=T_{s}[n]$ $+\int d^{3} r v_{s}(\mathbf{r}) n(\mathbf{r})$, this implies

$$
\frac{\delta F[n]}{\delta n(\mathbf{r})}=\mu-v_{\text {ext }}(\mathbf{r})
$$

and

$$
\frac{\delta T_{s}[n]}{\delta n(\mathbf{r})}=\mu-v_{s}(\mathbf{r}),
$$

where $T_{S}[n]$ is the noninteracting kinetic energy functional, $F[n]=T[n]+U[n]$ is the internal energy functional, expressed in terms of the interacting kinetic energy $T[n]$ and the interaction energy $U[n], \mu$ is the chemical potential, $v_{\text {ext }}(\mathbf{r})$ is the external potential and $v_{s}(\mathbf{r})$ the effective KS potential.

Both the effective and the external potential are only defined up to a constant, which does not change the form of the eigenfunctions. Consider now a gapped open system, connected to a particle reservoir with fixed chemical potential initially in the gap, and gradually change the constant. As long as the change is sufficiently small, the chemical potential remains in the gap, the density $n(\mathbf{r})$ does not change, and the derivatives on the left-hand side of Eqs. (12) and (13) change continuously.

However, once the change in the constant is large enough to affect the number of occupied levels, the situation changes: as soon as a new level falls below the chemical potential, or emerges above it, the number of particles in the system changes discontinuously by an integer, and the chemical potential adjusts itself to the new total particle number. For later convenience we call the two values of $\mu$ on the left and the right of integer particle number $\mu_{-}$and $\mu_{+}$, respectively.

When the right-hand side of Eqs. (12) and (13) changes discontinuously, the left-hand side must also change discontinuously. This means that the functional derivatives of $F[n]$ and $T_{s}[n]$ change discontinuously for variations $\delta n(\mathbf{r})$ such that $N$ passes through an integer, and are not defined precisely at the integer. We can also argue conversely that if the functional derivatives existed at all $n(\mathbf{r})$ they would determine the potentials uniquely. Since the potentials are unique only up to a constant, the functional derivatives cannot exist for the density variations $\delta n$ arising from changing the potential by a constant. In a gapped system, these are the $\delta n$ integrating to an integer.

Either way, we see that the indeterminacy of the potentials with respect to a constant implies that the functionals $F[n]$ and $T_{s}[n]$ display derivative discontinuities for certain directions in density space along which the total particle number changes by an integer. This is the famous integer discontinuity of DFT. ${ }^{5-7}$

\section{Connection of discontinuities and gaps: ensemble DFT}

Up to this point we have defined $\Delta_{x c}$ as a many-body correction to the single-particle gap, and deduced the existence of derivative discontinuities from noting the nonuniqueness of the external potentials with respect to a constant. These two conceptually distinct phenomena are related by ensemble DFT for systems with fractional particle number, describing open systems in contact with a particle reservoir. ${ }^{5-7}$ For such systems ensemble DFT guarantees that the ground-state energy as a function of particle number, $E(N)$, is a set of straight lines connecting values at integer particle numbers.

For straight lines, the derivative at any $N$ can be obtained from the values at the end points,

$$
-A=E(N+1)-E(N)=\left.\frac{\partial E}{\partial N}\right|_{N+\delta N}=\mu_{+}=\left.\frac{\delta E}{\delta n(\mathbf{r})}\right|_{N+\delta N}
$$

and

$$
-I=E(N)-E(N-1)=\left.\frac{\partial E}{\partial N}\right|_{N-\delta N}=\mu_{-}=\left.\frac{\delta E}{\delta n(\mathbf{r})}\right|_{N-\delta N} .
$$

The many-body fundamental gap is thus the derivative discontinuity of the total energy across densities integrating to an integer,

$$
E_{g}=I-A=\left.\frac{\delta E[n]}{\delta n(\mathbf{r})}\right|_{N+\delta N}-\left.\frac{\delta E[n]}{\delta n(\mathbf{r})}\right|_{N-\delta N} .
$$

This energy functional is commonly written as $E=T_{s}+V$ $+E_{H}+E_{x c}$, where the external potential energy $V$ and the Hartree energy $E_{H}$ are manifestly continuous functionals of the density. Hence, the energy gap reduces to the sum of the discontinuity of the noninteracting kinetic energy $T_{s}$ and that of the $x c$ energy $E_{x c}$.

The entire argument up to this point can be repeated for a noninteracting system in external potential $v_{s}$. The energy of this system is $E_{K S}=T_{s}+V_{s}$, of which only the first term can be discontinuous. Hence the fundamental gap of the KS system is given by the discontinuity of $T_{s}$, 


$$
E_{g, K S}=\left.\frac{\delta T_{S}[n]}{\delta n(\mathbf{r})}\right|_{N+\delta N}-\left.\frac{\delta T_{S}[n]}{\delta n(\mathbf{r})}\right|_{N-\delta N} .
$$

Returning now to the many-body gap, written as the sum of the discontinuities of $T_{s}$ and $E_{x c}$, we arrive at

$$
E_{g}=E_{g, K S}+\left.\frac{\delta E_{x c}[n]}{\delta n(\mathbf{r})}\right|_{N+\delta N}-\left.\frac{\delta E_{x c}[n]}{\delta n(\mathbf{r})}\right|_{N-\delta N},
$$

or, by means of Eq. (9),

$$
\Delta_{x c}=\left.\frac{\delta E_{x c}[n]}{\delta n(\mathbf{r})}\right|_{N+\delta N}-\left.\frac{\delta E_{x c}[n]}{\delta n(\mathbf{r})}\right|_{N-\delta N} .
$$

This identifies the $x c$ correction to the single-particle gap as the derivative discontinuity arising from the nonuniqueness of the potentials with respect to an additive constant..$^{5-7}$

Importantly, this connection is not required to define the $x c$ corrections and neither is its existence enough to conclude that these corrections are nonzero. Many-body corrections to the single-particle gap can be defined independently of any particular property of the density functional (or even without using any density-functional theory), and whether for a given system these corrections are nonzero or not depends on the electronic structure of that particular system, and does not follow from the formal possibility of a derivative discontinuity, because this discontinuity itself might be zero. Thus, the question of the existence and size of $x c$ corrections to the charge gap must be asked for each system anew. As we will see in the next section, the same is true for the spin gap.

\section{SPIN GAP}

We have provided the above rather detailed summary of the definition of the fundamental charge gap and its connection to nonuniqueness and to derivative discontinuities to prepare the ground for the following discussion of the spin gap. In order to arrive at a consistent DFT definition of spin gaps, we follow the steps outlined in the charge case: (i) define appropriate gaps and their $x c$ corrections, (ii) use the nonuniqueness of the SDFT potentials to show the existence of spin derivative discontinuities, and (iii) identify a suitable spin ensemble to connect the two.

\section{A. Spin gap vs charge gap}

To introduce a spin gap or a spin-flip energy (see below for precise definitions) we consider processes in which only the total spin of the system is changed, while the particle number remains the same. There cannot be any definition in terms of particle addition and removal energies, since in these processes the charge changes, too, which is not what one wants the spin gap to describe. In other words, the change of quantum numbers related to a spin flip is \pm 1 for the spin and 0 for charge. Note that this is an excitation energy, where the excitation takes place under the constraints of constant particle number and change of total spin by one unit. This is the key difference to the previous section, from which all other differences follow.

\section{B. Definition of spin gaps: spin-flip energies and spin stiffness}

First, we define the spin up-flip energy and the spin downflip energy in terms of many-body energies as

$$
\begin{aligned}
& E^{s f+}(N)=E(N, S+1)-E(N, S), \\
& E^{s f-}(N)=E(N, S-1)-E(N, S) .
\end{aligned}
$$

Here $E(N, S)$ is the lowest energy in the $N$-particle spin- $S$ subspace, where $S$ is the eigenvalue of the $z$ component of the total spin, and we assumed that spin up and spin down are good quantum numbers. This implies, in particular, that spin-orbit coupling is excluded from our analysis. (Of course these definitions only apply if the respective flips are actually possible; in other words, if $S$ does not yet have the maximal or minimal value for a given $N$.) The energy differences in Eqs. (20) and (21) can be estimated in a $\triangle S C F$-like way by performing approximate, say LDA, calculations of total energies maintaining the occupation of the KS levels fixed such as to reproduce total spin $S, S+1$ and $S-1$, respectively. Interestingly, these differences are also connected rigorously to a difference of KS eigenvalues at $S=1$ corrected by suitable derivative discontinuities, and can be obtained from time-dependent DFT or from ensemble DFT as we will show below.

Conceptually, the differences $E^{s f+}(N)$ and $E^{s f-}(N)$ are similar to the concepts of affinity and ionization energy, Eqs. (2) and (3). However, affinities and ionization energies are always defined with the smaller value (of $N$ ) as the first term in the differences, whereas spin-flip energies are conventionally defined as final state minus initial state, i.e., both spinflip energies measure an energy cost. Therefore, the downflip is the spin counterpart to the ionization energy, while the up-flip is the spin counterpart to minus the electron affinity.

A more important difference is that the spin-flip energies involve excited-state energies $E(N, S+1)$ and $E(N, S-1)$ of the $N$-particle system, instead of ground-state energies, and in this sense are more similar to the optical gap in the charge case than to the quantities used in evaluating the fundamental gap. Alternatively, these energies can also be considered ground-state energies for sectors of Hilbert space restricted to a given total $S$, which by adding a suitable constant can be made into absolute ground-state energies, but we will not make use of this alternative interpretation in the following.

KS spin-flip energies are related analogously to singleparticle eigenvalues, according to

$$
\begin{aligned}
& E_{K S}^{s f+}=\epsilon_{l(\uparrow)}-\epsilon_{H(\downarrow)}, \\
& E_{K S}^{s f-}=\epsilon_{l(\downarrow)}-\epsilon_{H(\uparrow)},
\end{aligned}
$$

where all energies are calculated at the same $N, S$. Here $l(\sigma)$ means the lowest unoccupied spin $\sigma$ state, and $H(\sigma)$ means the highest occupied spin $\sigma$ state. Similarly, $L(\sigma)$ and $h(\sigma)$ denote the lowest occupied spin $\sigma$ state and $h(\sigma)$ the highest unoccupied spin $\sigma$ state, respectively. This notation is nonstandard, but helpful, and further illustrated in Fig. 1.

In the same way, we can also define the spin conserving $(s c)$ single-particle gaps in each spin channel, as 

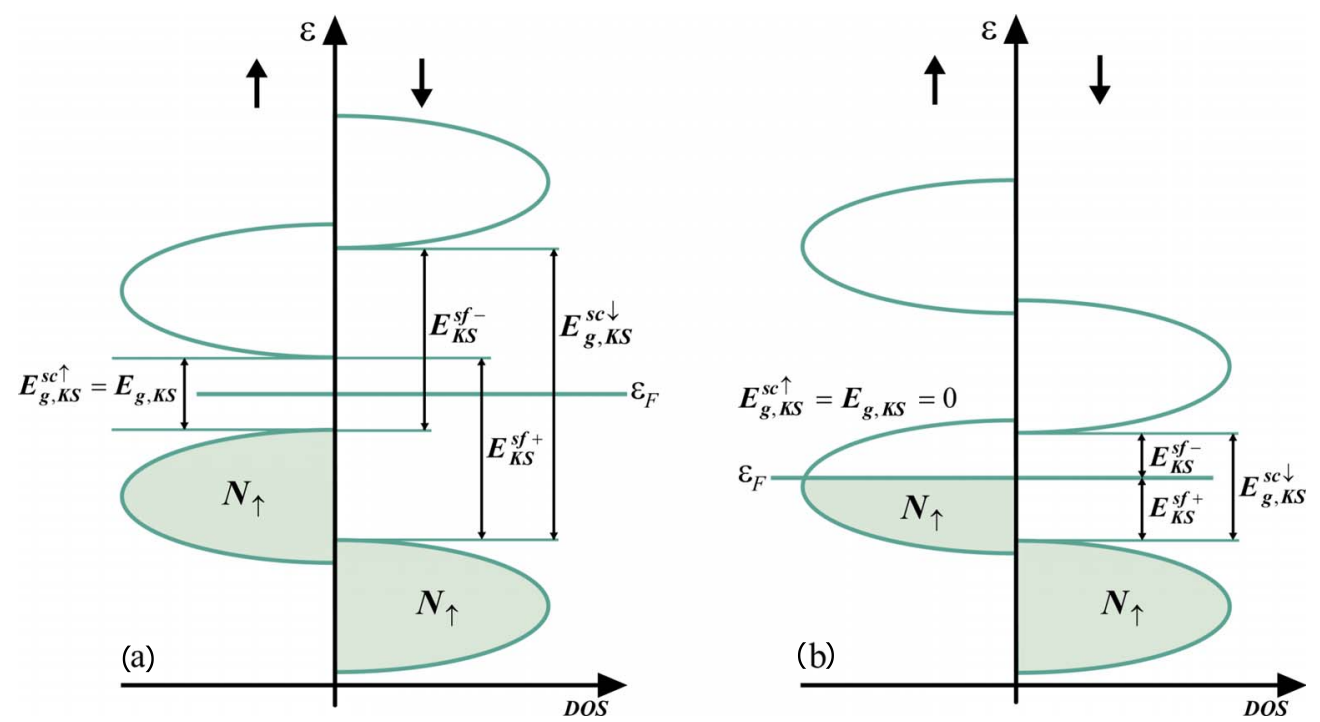

FIG. 1. (Color online) (a) spin-resolved single-particle (KS) density-of-states of a spin-polarized insulator. Two spin-flip energies and two spin-conserving gaps can be defined. (b) the half-metallic ferromagnet is a special case in which the gap in one spin channel (say spin up) is zero. In this case, there is only one spin-conserving gap, equal to the sum of both spin-flip energies, $E_{K S}^{s f-}+E_{K S}^{s f+}=E_{s, K S}^{s c}$, and the KS charge gap is zero, due to the presence of the gapless spin down channel. Figure 1 courtesy of Daniel Vieira.

$$
\begin{aligned}
& E_{g, K S}^{s c, \uparrow}=\epsilon_{l(\uparrow)}-\epsilon_{H(\uparrow)}, \\
& E_{g, K S}^{s c, \downarrow}=\epsilon_{l(\downarrow)}-\epsilon_{H(\downarrow)} .
\end{aligned}
$$

The spin-conserving gaps and the spin-flip energies are necessarily related by $E_{g, K S}^{s c \uparrow}+E_{g, K S}^{s c \downarrow}=E_{K S}^{s f-}+E_{K S}^{s f+}$. A look at Fig. 1 clarifies these definitions. If the system is non-spin-polarized, both spin-flip energies and both spin-conserving gaps become equal to the ordinary KS charge gap, which in our present notation reads $\epsilon_{l}-\epsilon_{H}$.

In the same way as for charge gaps, we can now also consider the sum and the difference of the spin-flip energies. The sum

$$
E_{s}=E^{s f-}+E^{s f+}
$$

of the energies it costs to flip a spin up and a spin down is formally analogous to the fundamental gap (1), but with the important difference that $E_{s}$ involves excited-state energies. The unusual sign (sum instead of difference) arises simply because both spin-flip energies measure costs, whereas the affinity featured in Eq. (1) measures an energy gain.

The formal analogy to Eq. (1) suggests that the quantity defined in Eq. (26) be called the fundamental spin gap. In practice, however, the name spin gap is more appropriately applied to the individual spin-flip energies. The physical interpretation of their sum, Eq. (26), is revealed by expressing it in terms of the many-body energies by means of Eqs. (20) and (21),

$$
E_{s}=E(N, S+1)+E(N, S-1)-2 E(N, S) .
$$

This is of the form of a discretized second derivative $\partial^{2} E(N, S) / \partial S^{2}$, which identifies $E_{S}$ as the discretized spin stiffness [we anticipated this interpretation when attaching a subscript $s$ for stiffness to the sum in Eq. (26)]. We note that half of $I-A$ is known in quantum chemistry as chemical hardness, which conveys a very similar idea as stiffness. Generically, we refer to all three quantities $E^{s f-}, E^{s f+}$, and $E_{s}$ as spin gaps.

The spin electronegativity can be defined as half of the difference of the spin-flip energies, $\chi^{s}=\left(E^{s f-}-E^{s f+}\right) / 2$. This quantity has the following interpretation: if $\chi^{s}>0$, it costs less energy to flip a spin up than to flip a spin down, whereas if $\chi^{s}<0$ the down flip is energetically cheaper.

The KS spin stiffness is defined as the sum of KS spin-flip energies,

$$
E_{s, K S}=E_{K S}^{s f-}+E_{K S}^{s f+},
$$

or, with Eqs. (22) and (23),

$$
E_{s, K S}=\left[\epsilon_{l(\downarrow)}-\epsilon_{H(\uparrow)}\right]+\left[\epsilon_{l(\uparrow)}-\epsilon_{H(\downarrow)}\right] .
$$

This is analogous to Eq. (5), except that in spin flips nothing is removed to infinity or brought in from infinity. Thus, differently from the KS ionization energy and electron affinity, the spin-flip energies require two single-particle energies for their definition instead of one, and in contrast with the KS charge gap the KS spin stiffness requires four single-particle energies instead of two.

This missing analogy is physically meaningful: conventional gaps are defined in terms of particle addition and removal processes and are ground-state properties. To define pure spin gaps (i.e., spin-flip energies and spin stiffness) in which the charge does not change, we cannot make use of particle addition and removal processes but have to use spin flip processes instead. However, spin flips are excitation energies, and we must specify both initial and final states to define them properly.

We also note that the many-body spin stiffness has no simple expression in terms of eigenvalues which would be analogous to Eq. (8). Such an expression would require the spin counterpart to Koopmans' theorem $I(N) \equiv I_{K S}(N)$, which 
TABLE I. Kohn-Sham energy eigenvalues (in eV) for the Lithium atom. The $1 s \uparrow, 2 s \uparrow$ and $1 s \downarrow$ levels are occupied. KS: energy eigenvalues obtained by inversion from quasiexact densities. XX denotes exact exchange (Ref. 12), and KLI is the Krieger-LiIafrate approximation (Ref. 13).

\begin{tabular}{lrrrrr}
\hline \hline & \multicolumn{1}{c}{$\mathrm{KS}^{\mathrm{a}}$} & \multicolumn{1}{c}{$\mathrm{KS}^{\mathrm{b}}$} & \multicolumn{1}{c}{$\mathrm{XX}^{\mathrm{a}}$} & $\mathrm{KLI}-\mathrm{XX}$ & \multicolumn{1}{c}{ LSDA } \\
\hline$-\epsilon_{1 s \uparrow}$ & 55.97 & 58.64 & 55.94 & 56.64 & 51.02 \\
$-\epsilon_{2 s \uparrow}$ & 5.39 & 5.39 & 5.34 & 5.34 & 3.16 \\
$-\epsilon_{2 p \uparrow}$ & 3.54 & & 3.48 & 3.50 & 1.34 \\
$-\epsilon_{1 s \downarrow}$ & 64.41 & 64.41 & 67.18 & 67.14 & 50.81 \\
$-\epsilon_{2 s \downarrow}$ & 8.16 & 5.87 & 8.25 & 8.23 & 2.09 \\
\hline \hline
\end{tabular}

Reference 12 .

${ }^{b}$ Reference 14 .

is not available for spin-flip energies. Hence, in general both spin-flip energies $E^{s f-}$ and $E^{s f+}$ may be individually different from their KS counterparts $E_{K S}^{s f-}$ and $E_{K S}^{s f+}$,

$$
\begin{aligned}
& E^{s f-}=E_{K S}^{s f-}+\Delta_{x c}^{s f-}, \\
& E^{s f+}=E_{K S}^{s f+}+\Delta_{x c}^{s f+} .
\end{aligned}
$$

We can, moreover, establish a relation between the manybody spin stiffness and the KS spin stiffness by rewriting the former as

$$
E_{s}=E_{s, K S}+\Delta_{x c}^{s}=E_{K S}^{s f-}+E_{K S}^{s f+}+\Delta_{x c}^{s},
$$

which defines $\Delta_{x c}^{s}$ as the $x c$ correction to the KS spin stiffness. The important thing to notice in Eq. (32) is that there is no reason to attribute $\Delta_{x c}^{s}$ only to the up-flip energy. This is a key difference to the charge case, where $I(N) \equiv I_{K S}(N)$ and the $x c$ correction could thus be attributed only to the electron affinity. Rather, the spin-flip corrections are connected by

$$
\Delta_{x c}^{s}=\Delta_{x c}^{s f-}+\Delta_{x c}^{s f+} .
$$

\section{Example: the Li atom}

To give an explicit example of the quantities introduced in the previous section, we now consider the Li atom. For this system, KS eigenvalues $\epsilon_{H \uparrow}=\epsilon_{2 s \uparrow}, \epsilon_{l \uparrow}=\epsilon_{2 p \uparrow}, \epsilon_{l \downarrow}=\epsilon_{2 s \downarrow}$, and $\epsilon_{H \downarrow}=\epsilon_{1 s \downarrow}$ have been obtained by numerical inversion of the KS equation starting from near-exact densities (see Table I). ${ }^{12,14}$

The KS spin-flip energies are obtained as

$$
\begin{aligned}
& E_{K S}^{s f+}=\epsilon_{2 p \uparrow}-\epsilon_{1 s \downarrow}, \\
& E_{K S}^{s f-}=\epsilon_{2 s \downarrow}-\epsilon_{2 s \uparrow} .
\end{aligned}
$$

They are given in Table II, together with the spin stiffness $E_{s}$, see Eq. (26). Table II also presents the corresponding experimental many-body energy differences for the Li atom, which were obtained using spectroscopic data for the lowest quartet state ${ }^{4} P^{0}$ and accurate wave-function based theory. ${ }^{15,16}$ Relativistic effects and other small corrections included in the experimental data are ignored since they are too small on the scale of energies we are interested in.

Table II also gives the $x c$ corrections to the single-particle spin flip energies, see Eqs. (30) and (31), and the $x c$ correction to the spin stiffness, $\Delta_{x c}^{s}$, see Eq. (32). As a consistency test we verified that relation (33), which connects the $x c$ corrections of the spin-flip energies to the $x c$ corrections of the spin stiffness, is satisfied.

We also carried out calculations using the exact-exchange (XX) eigenvalues of Ref. 12 in order to separately assess the size of exchange and correlation effects. The resulting value of $\Delta_{x}^{s}=-3.38 \mathrm{eV}$ indicates a larger (more negative) correction than in the calculation including correlation. An approximate KLI-XX calculation ${ }^{13}$ yields very similar results, while the LSDA data are completely different and do not even reproduce the correct sign.

Three of the required "exact" KS single-particle eigenvalues are also reported in Ref. 14 (we use the result of Ref. 12 for the missing value of $\epsilon_{2 p \uparrow}$ ). The value of $\epsilon_{2 s \downarrow}$ is quite different than the value reported in Ref. 12 (-5.87 eV versus

TABLE II. Single-particle spin-flip energies [Eqs. (34) and (35)] and spin stiffness [Eq. (29)], their experimental (Expt.) counterparts, Eqs. (20), (21), and (26), and the resulting $x c$ corrections defined in Eqs. (30)-(32), for the lithium atom. In the columns labeled KS we employ KS eigenvalues obtained from near-exact densities, while in the columns labeled XX, KLI-XX, and LSDA we use approximate eigenvalues obtained from standard SDFT calculations. The experimental values were obtained using spectroscopic data for the lowest quartet state ${ }^{4} P^{0}$ from Ref. 15 as well as accurate wave-function based theory from Ref. 16. All values are in $\mathrm{eV}$.

\begin{tabular}{lcccccc}
\hline \hline & $\mathrm{KS}^{\mathrm{a}}$ & $\mathrm{KS}^{\mathrm{b}}$ & $\mathrm{XX}^{\mathrm{a}}$ & KLI-XX & LSDA & Expt. \\
\hline$E^{s f+}$ & 60.87 & 60.87 & 63.70 & 63.64 & 49.47 & 57.41 \\
$E^{s f-}$ & -2.77 & -0.48 & -2.91 & -2.89 & 1.07 & 0 \\
$E_{s}$ & 58.10 & 60.39 & 60.79 & 60.75 & 50.54 & 57.41 \\
& & & & & & \\
$\Delta_{x c}^{s f+}$ & -3.46 & -3.46 & -6.29 & -6.23 & 7.94 & \\
$\Delta_{x c}^{s f-}$ & 2.77 & 0.48 & 2.91 & 2.89 & -1.07 & \\
$\Delta_{x c}^{s}$ & -0.69 & -2.98 & -3.38 & -3.34 & 6.87 & \\
\hline \hline
\end{tabular}

${ }^{\text {aReference } 12 .}$

${ }^{\mathrm{b}}$ Reference 14 , taking $-\epsilon_{2 p \uparrow}=3.54 \mathrm{eV}$ from Reference 12 . 
-8.16 eV), and consequently we obtain a rather different value of $\Delta_{x c}^{s}(-2.98 \mathrm{eV}$ versus $-0.69 \mathrm{eV})$. Nevertheless, both sets of data sustain our main conclusions in this section:

(i) Simple LSDA calculations give rise to serious qualitative errors. As can be seen from Table II, one obtains spinflip energies that are drastically too small $\left(E^{s f+}\right)$ or have the wrong sign $\left(E^{s f-}\right)$. The resulting $x c$ corrections also suffer from having the wrong sign. These shortcomings of the LSDA are hardly surprising in view of its well-established failure to describe the charge gap. Although the nature of the correction required for charge gaps is different from that required for spin gaps, both must compensate for the basic deficiencies of their common starting point (the LDA eigenvalues): the fact that LDA has no discontinuity whatsoever and that it predicts the wrong asymptotics for the KS potential of finite systems.

(ii) Even the precise KS eigenvalues do not predict the exact spin flip energies and spin stiffness, i.e., the $x c$ corrections introduced in Sec. III B on purely formal grounds are indeed nonzero. The absolute size of these corrections implies that a simple KS eigenvalue calculation of spin gaps can be seriously in error.

(iii) Exchange-only calculations overestimate (in modulus) the size of the gap corrections. This implies that there is substantial cancellation between the exchange and the correlation contribution to the full correction. This is the same trend known for charge gaps.

(iv) The $x c$ corrections to both the up-flip energy and the spin stiffness turn out to be negative; in other words the KS calculation overestimates these quantities. This is the opposite of what occurs in the case of the fundamental charge gap, which is underestimated by the KS calculation. We note that hints of an overestimation of the experimental spin-flip energies by $\mathrm{KS}$ eigenvalue differences have also been observed for half-metallic ferromagnets. In the case of $\mathrm{CrO}_{2}$, for example, Ref. 17 reports experimental spin-flip energies in the range 0.06 to $0.25 \mathrm{eV}$ and compiles SDFT predictions that range from 0.2 to $0.7 \mathrm{eV}$ (and in one case even $1.7 \mathrm{eV}$ ).

\section{Nonuniqueness and derivative discontinuities in SDFT}

Above we pointed out that the effective and external potentials of DFT are determined by the ground-state density up to an additive constant. However, this statement only holds when one formulates DFT exclusively in terms of the charge density, as we have done in discussing charge gaps. It does not hold when one works with spin densities, as in SDFT, or current densities, as in current-DFT (CDFT).

In these cases the densities still determine the wave function, but they do not uniquely determine the corresponding potentials. A first example of this nonuniqueness problem of generalized DFTs was already encountered in early work on SDFT, for the single-particle KS Hamiltonian. ${ }^{18}$ Later, this observation was extended to the SDFT many-body Hamiltonian, ${ }^{19,20}$ and further examples were obtained in CDFT (Ref. 21) and SDFT on lattices. ${ }^{22}$

Nonuniqueness is a generic feature of generalized (multidensity) DFTs, consequences of which are still under investigation. ${ }^{23-27}$ In particular, Refs. 19 and 20 already point out that the nonuniqueness of the potentials of SDFT implies that the SDFT functionals can have additional derivative discontinuities, because, if the functional derivatives of $F$ and $T_{s}$ in multidensity DFTs such as SDFT and CDFT existed for all densities, they would determine the corresponding potentials uniquely. Very recently, Gál and collaborators ${ }^{27}$ pointed out that one-sided derivatives may still exist, and explored consequences of this for the DFT description of chemical reactivity indices, while Perdew and Sagvolden ${ }^{28}$ and Chan ${ }^{29}$ discussed the extension of the classic work on derivative discontinuities within DFT (Refs. 5-7) to SDFT.

Just as in the charge case, derivative discontinuities result from the nonuniqueness of the spin-dependent potentials, while corrections to single-particle gaps result from the auxiliary nature of Kohn-Sham eigenvalues. In the charge case, both distinct phenomena could be connected by means of ensemble DFT for systems of fractional-particle number. The question then arises if a similar connection can also be established in the spin case. This requires an investigation of spin ensembles.

\section{E. Spin ensembles}

Consequences of the nonuniqueness of the potentials of SDFT for the calculation of spin gaps were already hinted at in Refs. 19 and 20, where it was pointed out that there may be a spin-gap problem in SDFT similarly to the well known band-gap problem of DFT.

To make these hints more precise, we first recall, from the above, that the quantity usually called the spin gap is actually what we here called the spin-flip gap, and is analogous to the ionization energy or the electron affinity in the charge case, not to the fundamental particle gap. The spin-dependent quantity that is most analogous to the fundamental particle gap is the discretized spin stiffness of Eqs. (26) and (27). However, regardless of whether one focuses on the spin-flip energies or on the spin stiffness, the spin situation is not completely analogous to the charge situation because both the spin-flip gaps and the spin stiffness are defined in terms of excited states of an $N$-particle Hamiltonian, while charge gaps are defined in terms of ground-state energies of Hamiltonians with different particle numbers.

To identify a suitable ensemble, we write the energy associated with a generic ensemble of two systems, A and B, as

$$
E^{w}=(1-w) E_{A}+w E_{B},
$$

where $0 \leq w \leq 1$ is the ensemble weight. If $\mathrm{A}$ and $\mathrm{B}$ have different particle numbers, $N_{A}$ and $N_{B}=N_{A} \pm 1$, this becomes the usual fractional-particle number ensemble, which is unsuitable for our present investigation where the involved systems differ in the spin but not the charge quantum numbers.

A spin-dependent ensemble was recently constructed by Yang and collaborators ${ }^{30,31}$ in order to understand the static correlation error of common density functionals. In this spin ensemble, A and B have different (possibly fractional) spin, but are degenerate in energy. The constancy condition, whose importance and utility is stressed in Refs. 30 and 31, arises directly from the restriction of the ensemble to degenerate states. While useful for the purposes of analyzing the 
static correlation error, this spin ensemble is too restrictive for our purposes, as it excludes the excited states involved in the definition of spin-flip gaps and of the spin stiffness.

Ensembles involving excited states have been employed in DFT in connection with the calculation of excitation energies. ${ }^{32-34}$ Here $\mathrm{A}$ and $\mathrm{B}$ differ in energy but stem from the same Hamiltonian, with fixed particle number. Excitedstate ensemble theory leads to a simple expression relating the first excitation energy to a KS eigenvalue difference, ${ }^{33}$

$$
E_{B}-E_{A}=\epsilon_{M+1}^{w}-\epsilon_{M}^{w}+\left.\frac{\partial E_{x c}^{w}[n]}{\partial w}\right|_{n=n_{w}},
$$

where $E_{B}$ and $E_{A}$ are the energies of first excited and the ground state of the many-body system, respectively, $\epsilon_{M+1}^{w}$ and $\epsilon_{M}^{w}$ are the highest occupied and lowest unoccupied KS eigenvalues, and $E_{x c}^{w}$ is the ensemble $x c$ functional. Equation (37) holds for ensemble weights in the range $0 \leq w \leq 1 / 2$. Levy showed ${ }^{35}$ that the last term in this equation is related to a derivative discontinuity according to

$$
\left.\frac{\partial E_{x c}^{w}[n]}{\partial w}\right|_{n=n^{w}}=\left.\frac{\delta E_{x c}^{w=0}[n]}{\delta n(\mathbf{r})}\right|_{n=n^{w=0}}-\left.\frac{\delta E_{x c}^{w}[n]}{\delta n(\mathbf{r})}\right|_{n=n^{w}}
$$

for $w \rightarrow 0$. Here $n^{w}=(1-w) n_{A}+w n_{B}$ is the ensemble density, and the discontinuity arises because even in the $w \rightarrow 0$ limit the ensemble density does contain an admixture of the state B with energy $E_{B}>E_{A}$ and thus decays differently from $n_{0}$ as $r \rightarrow \infty .{ }^{35}$ Levy developed his argument explicitly only for the spin-unpolarized case, but already pointed out in the original paper that the results carry over to spin-polarized situations.

In our case, we take A to be the ground state and B to be the lowest-lying state differing from it by a spin flip. To be specific, let us assume that the spin is flipped up. In this case we obtain from Eqs. (37) and (38) in the limit $w \rightarrow 0$, and using our present notation,

$$
\begin{aligned}
E^{s f+}(N)= & E(N, S+1)-E(N, S) \\
= & \epsilon_{l(\uparrow)}^{w}-\epsilon_{H(\downarrow)}^{w}+\left.\frac{\partial E_{x c}^{w}\left[n_{\uparrow}, n_{\downarrow}\right]}{\partial w}\right|_{\substack{n_{\uparrow}=n_{\uparrow}^{w} \\
n_{\downarrow}=n_{\downarrow}^{w}}} \\
= & \epsilon_{l(\uparrow)}^{w}-\epsilon_{H(\downarrow)}^{w}+\left.\frac{\delta E_{x c}^{w=0}\left[n_{\uparrow}, n_{\downarrow}\right]}{\delta n_{\downarrow}(\mathbf{r})}\right|_{\substack{n_{\uparrow}=n_{\uparrow}^{w=0} \\
n_{\downarrow}=n_{\downarrow}^{w=0}}} \\
& -\left.\frac{\delta E_{x c}^{w}\left[n_{\uparrow}, n_{\downarrow}\right]}{\delta n_{\downarrow}(\mathbf{r})}\right|_{n_{\uparrow}=n_{\uparrow}^{w}} \\
= & E_{w, K S}^{s f+}(N)+n_{\downarrow}^{w} \\
&
\end{aligned}
$$

for $w \rightarrow 0$. Equation (42), which is the ensemble version of our Eq. (31), illustrates that KS spin-flip excitations, too, acquire a many-body correction arising from a derivative discontinuity.

In the particular case in which the spin flip costs no energy in the many-body and in the KS system, the preceding equation reduces to $\Delta_{w, x c}^{s f+}=0$, which is the constancy condi- tion derived in Refs. 30 and 31 for spin ensembles of degenerate states.

We note that the KS eigenvalues and the discontinuity in Eqs. (40)-(42) must be evaluated by taking the $w \rightarrow 0$ limit of the $w$-dependent quantities, while the quantities in Eq. (31) have no ensemble dependence. This complicates the evaluation of spin-flip energies and their discontinuities, as defined in Sec. III B, from ensemble DFT. Therefore, we turn to still another density-functional approach to excited states in order to evaluate these quantities: TDDFT.

\section{F. Connection to TDDFT}

TDDFT has established itself as the method of choice for calculating excitation energies in atomic and molecular systems, and is making rapid progress in nanoscale systems and solids as well. ${ }^{36,37}$ In this section we will make a connection between the preceding discussion and TDDFT, which will allow us to derive simple approximations for the $x c$ corrections to the single-particle spin-flip excitation energies and the spin stiffness.

To calculate the spin-conserving and the spin-flip excitation energies, it is necessary to use a noncollinear spindensity response theory, even if the system under study has a ground state with collinear spins (i.e., spin-up and -down with respect to the $z$ axis are good quantum numbers). In this way the spin-up and spin-down density responses can become coupled, and the description of spin-flip excitations (for instance, due to a transverse magnetic perturbation) becomes possible. In TDDFT, the spin-conserving and the spinflip excitation energies can be obtained from the following eigenvalue equations, which are a generalization of the widely used Casida equations ${ }^{38}$ for systems with noncollinear spin: ${ }^{39}$

$$
\begin{aligned}
& \sum_{\sigma \sigma^{\prime} i^{\prime} a^{\prime}} \sum_{i}\left\{\left[\delta_{i^{\prime} i} \delta_{a^{\prime} a} \delta_{\sigma \alpha} \delta_{\sigma^{\prime} \alpha^{\prime}} \omega_{a^{\prime} \sigma^{\prime} i^{\prime} \sigma}+K_{i \alpha a \alpha^{\prime}, i^{\prime} \sigma a^{\prime} \sigma^{\prime}}^{\alpha \alpha^{\prime}, \sigma \sigma^{\prime}}\right] X_{i^{\prime} \sigma a^{\prime} \sigma^{\prime}}\right. \\
& \left.+K_{i \alpha a \alpha^{\prime}, i^{\prime} \sigma a^{\prime} \sigma^{\prime}}^{\alpha \alpha^{\prime}, \sigma^{\prime} \sigma} Y_{i^{\prime} \sigma, a^{\prime} \sigma^{\prime}}\right\}=-\omega X_{i \alpha, a \alpha^{\prime}}, \\
& \sum_{\sigma \sigma^{\prime} i^{\prime} a^{\prime}}\left\{K_{i \alpha a \alpha^{\prime}, i^{\prime} \sigma a^{\prime} \sigma^{\prime}}^{\alpha^{\prime} \alpha, \sigma \sigma^{\prime}} X_{i^{\prime} \sigma a^{\prime} \sigma^{\prime}}+\left[\delta_{a^{\prime} a} \delta_{i^{\prime} i} \delta_{\sigma^{\prime} \alpha^{\prime}} \delta_{\sigma \alpha} \omega_{a^{\prime} \sigma^{\prime} i^{\prime} \sigma}\right.\right. \\
& \left.+K_{i \alpha a \alpha^{\prime}, i^{\prime} \sigma a^{\prime} \sigma^{\prime}}^{\alpha^{\prime} \alpha, \sigma^{\prime} \sigma} Y_{i^{\prime} \sigma, a^{\prime} \sigma^{\prime}}\right\}=\omega Y_{i \alpha, a \alpha^{\prime}},
\end{aligned}
$$

where we use the standard convention that $i, i$ and $a, a^{\prime}$ are indices of occupied and unoccupied KS orbitals, respectively, and $\alpha \alpha^{\prime}, \sigma \sigma^{\prime}$ are spin indices, and $\omega_{a^{\prime} \sigma^{\prime} i^{\prime} \sigma}=\epsilon_{a^{\prime} \sigma^{\prime}}$ $-\epsilon_{i^{\prime} \sigma}$. Choosing the KS orbitals to be real, without loss of generality, we have

$$
\begin{aligned}
K_{i \alpha a \alpha^{\prime}, i^{\prime} \sigma a^{\prime} \sigma^{\prime}}^{\alpha \alpha^{\prime}, \sigma \sigma^{\prime}}= & \int d^{3} r \int d^{3} r^{\prime} \psi_{i \alpha}(\mathbf{r}) \psi_{a \alpha^{\prime}}(\mathbf{r}) \\
& \times f_{\alpha \alpha^{\prime}, \sigma \sigma^{\prime}}^{H x c}\left(\mathbf{r}, \mathbf{r}^{\prime}, \omega\right) \psi_{i^{\prime} \sigma}\left(\mathbf{r}^{\prime}\right) \psi_{a^{\prime} \sigma^{\prime}}\left(\mathbf{r}^{\prime}\right) .
\end{aligned}
$$

Here, the subscript indices of the matrix elements $K$ refer to the KS orbitals in the integrand, and the superscript spin indices refer to the Hartree- $x c$ kernel 


$$
f_{\alpha, \alpha^{\prime}, \sigma \sigma^{\prime}}^{H x c}\left(\mathbf{r}, \mathbf{r}^{\prime}, \omega\right)=\frac{\delta_{\alpha \alpha^{\prime}} \delta_{\sigma \sigma^{\prime}}}{\left|\mathbf{r}-\mathbf{r}^{\prime}\right|}+f_{\alpha \alpha^{\prime}, \sigma \sigma^{\prime}}^{c c}\left(\mathbf{r}, \mathbf{r}^{\prime}, \omega\right),
$$

where the frequency-dependent $x c$ kernel is defined as the Fourier transform of the time-dependent $x c$ kernel

$$
f_{\alpha \alpha^{\prime}, \sigma \sigma^{\prime}}^{x c}\left(\mathbf{r}, t, \mathbf{r}^{\prime}, t^{\prime}\right)=\left.\frac{\delta v_{\alpha \alpha^{\prime}}^{x c}(\mathbf{r}, t)}{\delta n_{\sigma \sigma^{\prime}}\left(\mathbf{r}^{\prime}, t^{\prime}\right)}\right|_{\underline{n}(\mathbf{r}, t)=\underline{n}^{0}(\mathbf{r})} .
$$

Here, $\underline{n}(\mathbf{r}, t)$ and $\underline{n}^{0}(\mathbf{r})$ are the time-dependent and the ground-state $2 \times 2$ spin-density matrix, which follow from the DFT formalism for noncollinear spins. ${ }^{39-43}$

Equations (43) and (44) give, in principle, the exact spinconserving and spin-flip excitation energies of the system, provided the exact KS orbitals and energy eigenvalues are known, as well as the exact functional form of $f_{\alpha \alpha^{\prime}, \sigma \sigma^{\prime}}^{x c}$. We will now consider a simplified solution known as the singlepole approximation. ${ }^{44,45}$ It is obtained from the full system of Eqs. (43) and (44) by making the Tamm-Dancoff approximation (i.e., neglecting the off diagonals) and focusing only on the $H(\sigma) \rightarrow l\left(\sigma^{\prime}\right)$ excitations. In other words, we need to solve the $4 \times 4$ problem

$$
\sum_{\sigma \sigma^{\prime}}\left[\delta_{\sigma^{\prime} \alpha^{\prime}} \delta_{\sigma \alpha} \omega_{l \sigma^{\prime} H \sigma}+K_{H \alpha l \alpha^{\prime}, H \sigma l \sigma^{\prime}}^{\alpha^{\prime} \alpha, \sigma^{\prime} \sigma}\right] Y_{H \sigma, l \sigma^{\prime}}=\omega Y_{H \alpha, l \alpha^{\prime}} .
$$

For ground states with collinear spins, the only nonvanishing elements of the Hartree- $x c$ kernel are

$$
f_{\uparrow \uparrow, \uparrow \uparrow}^{H x c}, f_{\downarrow \downarrow, \downarrow \downarrow}^{H x c}, f_{\uparrow \uparrow, \downarrow \downarrow}^{H x c}, f_{\downarrow \downarrow, \uparrow \uparrow}^{H x c}, f_{\uparrow \downarrow, \uparrow \downarrow}^{x c}, f_{\downarrow \uparrow, \downarrow \uparrow}^{x c}
$$

(notice that there is no Hartree term in the spin-flip channel), and the spin-conserving and spin-flip excitation channels decouple into two separate $2 \times 2$ problems. For the spinconserving case, we have

$$
\operatorname{det}\left|\begin{array}{cc}
\omega_{\uparrow \uparrow}-\omega^{s c}+M_{\uparrow \uparrow, \uparrow \uparrow} & M_{\uparrow \uparrow, \downarrow \downarrow \downarrow} \\
M_{\downarrow \downarrow, \uparrow \uparrow} & \omega_{\downarrow \downarrow}-\omega^{s c}+M_{\downarrow \downarrow, \downarrow \downarrow}
\end{array}\right|=0,
$$

where we abbreviate $M_{\alpha \alpha^{\prime}, \sigma \sigma^{\prime}}=K_{H \alpha l \alpha^{\prime}, H \sigma l \sigma^{\prime}}^{\alpha^{\prime} \alpha, \sigma^{\prime} \sigma}(\omega)$ and $\omega_{\sigma^{\prime} \sigma}$ $=\omega_{l \sigma^{\prime}, H \sigma}=\epsilon_{l \sigma^{\prime}}-\epsilon_{H \sigma^{\prime}}$. From this, we get the two spinconserving excitation energies as

$$
\begin{aligned}
E^{s c \uparrow, \downarrow}= & \frac{\omega_{\uparrow \uparrow}+\omega_{\downarrow \downarrow}+M_{\uparrow \uparrow, \uparrow \uparrow}+M_{\downarrow \downarrow, \downarrow \downarrow}}{2} \\
& \pm\left[M_{\uparrow \uparrow, \downarrow \downarrow} M_{\downarrow \downarrow, \uparrow \uparrow}+\frac{1}{4}\left(\omega_{\uparrow \uparrow}-\omega_{\downarrow \downarrow}\right.\right. \\
& \left.\left.+M_{\uparrow \uparrow, \uparrow \uparrow}-M_{\downarrow \downarrow, \downarrow \downarrow}\right)^{2}\right]^{1 / 2},
\end{aligned}
$$

with the spin-conserving Kohn-Sham single-particle gaps $E_{g, K S}^{s c(\downarrow)}=\omega_{\uparrow \uparrow(\downarrow \downarrow)}$. The two spin-flip excitations follow immediately as

$$
\begin{aligned}
& E^{s f+}=\omega_{\uparrow \downarrow}+M_{\uparrow \downarrow, \uparrow \downarrow}, \\
& E^{s f-}=\omega_{\downarrow \uparrow}+M_{\downarrow \uparrow, \downarrow \uparrow},
\end{aligned}
$$

where $E_{K S}^{s f+}=\omega_{\uparrow \downarrow}$ and $E_{K S}^{s f-}=\omega_{\downarrow \uparrow}$.
This gives a simple approximation for the $x c$ correction to the spin stiffness,

$$
\Delta_{x c}^{s}=M_{\uparrow \downarrow, \uparrow \downarrow}+M_{\downarrow \uparrow, \downarrow \uparrow} .
$$

Explicit expressions for $f_{\alpha \alpha^{\prime}, \sigma \sigma^{\prime}}^{x c}$ can be obtained from the local spin-density approximation (LSDA), and we list them here for completeness (see also Wang and Ziegler ${ }^{39}$ ),

$$
\begin{gathered}
f_{\uparrow \uparrow, \uparrow \uparrow}^{x c}=\frac{\partial^{2}\left(n e_{\mathrm{xc}}^{h}\right)}{\partial n^{2}}+2(1-\zeta) \frac{\partial^{2} e_{\mathrm{xc}}^{h}}{\partial n \partial \zeta}+\frac{(1-\zeta)^{2}}{n} \frac{\partial^{2} e_{\mathrm{xc}}^{h}}{\partial \zeta^{2}}, \\
f_{\downarrow \downarrow, \downarrow \downarrow}^{x c}=\frac{\partial^{2}\left(n e_{\mathrm{xc}}^{h}\right)}{\partial n^{2}}-2(1+\zeta) \frac{\partial^{2} e_{\mathrm{xc}}^{h}}{\partial n \partial \zeta}+\frac{(1+\zeta)^{2}}{n} \frac{\partial^{2} e_{\mathrm{xc}}^{h}}{\partial \zeta^{2}}, \\
f_{\uparrow \uparrow, \downarrow \downarrow}^{x c}=\frac{\partial^{2}\left(n e_{\mathrm{xc}}^{h}\right)}{\partial n^{2}}-2 \zeta \frac{\partial^{2} e_{\mathrm{xc}}^{h}}{\partial n \partial \zeta}-\frac{\left(1-\zeta^{2}\right)}{n} \frac{\partial^{2} e_{\mathrm{xc}}^{h}}{\partial \zeta^{2}}, \\
f_{\uparrow \downarrow, \uparrow \downarrow}^{x c}=\frac{2}{n \zeta} \frac{\partial e_{\mathrm{xc}}^{h}(n, \zeta)}{\partial \zeta},
\end{gathered}
$$

where $f_{\downarrow \downarrow, \uparrow \uparrow}^{x c}=f_{\uparrow \uparrow, \downarrow \downarrow}^{x c}$ and $f_{\downarrow \uparrow, \downarrow \uparrow}^{x c}=f_{\uparrow \downarrow, \uparrow \downarrow}^{x c}$, and it is understood that all expressions are multiplied by $\delta\left(\mathbf{r}-\mathbf{r}^{\prime}\right)$ and evaluated at the local ground-state density and spin polarization, $n_{0}(\mathbf{r})$ $=n_{0 \uparrow}(\mathbf{r})+n_{0 \downarrow}(\mathbf{r})$ and $\zeta_{0}(\mathbf{r})=\left[n_{0 \uparrow}(\mathbf{r})-n_{0 \downarrow}(\mathbf{r})\right] / n_{0}(\mathbf{r})$. For the $x c$ energy density of the spin-polarized homogeneous electron gas we take the standard interpolation formula

$$
\begin{aligned}
e_{x c}^{h}(n, \zeta)= & e_{x c}^{h}(n, 0)+\frac{(1+\zeta)^{4 / 3}+(1-\zeta)^{4 / 3}-2}{2^{4 / 3}-2} \\
& \times\left[e_{x c}^{h}(n, 1)-e_{x c}^{h}(n, 0)\right] .
\end{aligned}
$$

The case of exact exchange (XX) in linear response can be treated exactly, though with considerable technical and numerical effort. ${ }^{46,47}$ A simplified expression of the XX $x c$ kernel was developed by Petersilka et al. ${ }^{44}$ and we have generalized their expression for the linear response of the spindensity matrix. We obtain

$$
f_{\uparrow \uparrow, \uparrow \uparrow}^{x}\left(\mathbf{r}, \mathbf{r}^{\prime}\right)=-\sum_{i, k}^{N_{\uparrow}} \frac{\psi_{k \uparrow}(\mathbf{r}) \psi_{k \uparrow}^{*}\left(\mathbf{r}^{\prime}\right) \psi_{i \uparrow}^{*}(\mathbf{r}) \psi_{i \uparrow}\left(\mathbf{r}^{\prime}\right)}{\left|\mathbf{r}-\mathbf{r}^{\prime}\right| n_{\uparrow}(\mathbf{r}) n_{\uparrow}\left(\mathbf{r}^{\prime}\right)}
$$

and similarly for $f_{\downarrow \downarrow, \downarrow}^{x}\left(\mathbf{r}, \mathbf{r}^{\prime}\right)$, and

$$
\begin{aligned}
f_{\uparrow \downarrow \uparrow \downarrow \downarrow}^{x}\left(\mathbf{r}, \mathbf{r}^{\prime}\right) & =-\sum_{i, k}^{N_{\uparrow}, N_{\downarrow}} \frac{\psi_{k \uparrow \uparrow}(\mathbf{r}) \psi_{k \uparrow}^{*}\left(\mathbf{r}^{\prime}\right) \psi_{i \downarrow}^{*}(\mathbf{r}) \psi_{i \downarrow}\left(\mathbf{r}^{\prime}\right)}{\left|\mathbf{r}-\mathbf{r}^{\prime}\right| \sqrt{n_{\uparrow}(\mathbf{r}) n_{\downarrow}(\mathbf{r}) n_{\uparrow}\left(\mathbf{r}^{\prime}\right) n_{\downarrow}\left(\mathbf{r}^{\prime}\right)}} \\
& =f_{\downarrow \uparrow, \downarrow \uparrow}^{x}\left(\mathbf{r}, \mathbf{r}^{\prime}\right) .
\end{aligned}
$$

Here, $N_{\uparrow}$ and $N_{\downarrow}$ are the number of occupied spin-up and spin-down orbitals.

We have evaluated Eqs. (51)-(54) for the spin-conserving and spin-flip excitation energies of the Lithium atom involving the $H(\sigma)$ and $l\left(\sigma^{\prime}\right)$ orbitals. The LSDA and KLI-XX orbital eigenvalues that are needed as input are given in Table I. 
TABLE III. Top part: lowest spin-conserving and spin-flip excitation energies for the lithium atom, calculated with LSDA and KLI-XX using differences of KS eigenvalues and TDDFT in the single-pole approximation (51)-(53). Bottom part: TDDFT $x c$ corrections to the KS spin-flip excitation energies, from Eqs. (52) and (53), and to the KS spin gap, Eq. (54). All numbers are in eV.

\begin{tabular}{|c|c|c|c|c|c|c|}
\hline & \multicolumn{2}{|c|}{ LSDA } & \multicolumn{2}{|c|}{ KLI-XX } & \multicolumn{2}{|c|}{ Exact } \\
\hline & $\mathrm{KS}$ & TDDFT & $\mathrm{KS}$ & TDDFT & $K S^{\mathrm{a}}$ & Expt. ${ }^{b}$ \\
\hline$E^{s c \uparrow}$ & 1.83 & 2.00 & 1.84 & 2.01 & 1.85 & 1.85 \\
\hline$E^{s c \downarrow}$ & 48.72 & 48.89 & 58.90 & 59.31 & 56.25 & 56.36 \\
\hline$E^{s f+}$ & 49.47 & 48.23 & 63.64 & 62.12 & 60.87 & 57.41 \\
\hline$E^{s f-}$ & 1.07 & 0.99 & -2.89 & -2.97 & -2.77 & 0.0 \\
\hline$\Delta_{x c}^{s f+}$ & \multicolumn{2}{|c|}{-1.24} & \multicolumn{2}{|c|}{-1.52} & \multicolumn{2}{|c|}{-3.46} \\
\hline$\Delta_{x c}^{s f-}$ & \multicolumn{2}{|c|}{-0.08} & \multicolumn{2}{|c|}{-0.07} & \multicolumn{2}{|c|}{+2.77} \\
\hline$\Delta_{x c}^{s}$ & \multicolumn{2}{|c|}{-1.32} & \multicolumn{2}{|c|}{-1.59} & \multicolumn{2}{|c|}{-0.69} \\
\hline
\end{tabular}

${ }^{a}$ Evaluated from the KS eigenvalues of Reference 12 .

${ }^{\mathrm{b}}$ Spectroscopic data from Ref. $48\left(E^{s c \uparrow, \downarrow}\right)$ and References 15 and $16\left(E^{s f+}\right)$.

The associated excitation energies are shown in Table III, where we compare KS excitations, i.e., differences of KS eigenvalues, with TDDFT excitations obtained using the single-pole approximation described above. All in all, the TDDFT excitation energies are not much improved compared to the KS orbital eigenvalue differences. The main reason is that the LSDA and KLI-XX KS energy eigenvalues are not particularly close to the exact KS energy eigenvalues, and furthermore that the single-pole approximation is too simplistic for this open-shell atom.

However, we observe that the $x c$ correction $\Delta_{x c}^{s}$ to the spin stiffness $E_{s}$, when directly calculated within LSDA or KLI-XX using the TDDFT formula (54), is reasonably close to the exact value, and has the correct sign. This tells us that, even though the KS spin gap itself may be not very good, the simple TDDFT expression (54) gives a reasonable approximation for the $x c$ correction to it.

The approximate $x c$ kernels used in this section are adiabatic, i.e., independent of frequency; however, our formalism is by no means restricted to the adiabatic approximation. The $\omega$ dependence of the $x c$ kernel plays a role in two key situations: for double (multiple) excitations, ${ }^{49}$ and for chargetransfer excitations involving open-shell fragments; ${ }^{50}$ neither is the case in our example. By definition, the spin gap only involves single spin flips, which rules out any contributions from double or multiple excitations. On the other hand, the spin gap of large (bio) molecular systems whose lowest spinflip excitations have charge-transfer character with openshell donor-acceptor fragments may indeed require a nonadiabatic $x c$ kernel.

\section{CONCLUSION}

The calculation of spin gaps and related quantities is important for phenomena like spin-flip excitations in finite systems, ${ }^{39}$ the magnetic and transport properties of extended systems such as half-metallic ferromagnets ${ }^{17}$ and, quite generally, in the emerging field of spintronics and spindependent transport.
Our aim in this paper was to show how to define and calculate spin gaps and related quantities from densityfunctional theory. The proper definition of spin gaps in SDFT is by no means obvious, and the straightforward extrapolation of concepts and properties from the charge case to the spin case is fraught with dangers. Therefore, we started our investigation by disentangling two aspects of the gap problem that in the charge case are usually treated together: the derivative discontinuity and the many-body correction to single-particle gaps.

On this background, we then provided a set of DFT-based definitions of quantities that are related to spin gaps, such as spin-conserving gaps, spin-flip gaps and the spin stiffness, pointing out in each case where possible analogies to the charge case exist, and when these analogies break down. In particular, spin-flips involve excitations, while particle addition and removal involves ground-state energies. As a consequence, single-particle spin-flip energies involve two eigenvalues (and not one) and single-particle spin gaps involve four (and not two). Moreover, each spin-flip energy may have its own $x c$ correction (there is no Koopmans' theorem for spin flips).

An evaluation of our definitions for the Lithium atom, making use of highly precise Kohn-Sham eigenvalues and spectroscopic data, shows that the many-body correction to spin gaps can indeed be nonzero. In fact, unlike what is common in the charge case, this correction turns out to be negative, i.e., the single-particle calculation overestimates the spin gap while it underestimates the charge gap. While this result for a single atom is consistent with available data on half-metallic ferromagnets, ${ }^{17}$ similar calculations must be performed for other systems before broad trends can be identified.

Next, we connected the many-body corrections to the spin gap and related quantities to ensemble DFT and to TDDFT. The former connection makes use of a suitable excited-state spin ensemble (different from the degenerate-state spin ensemble recently proposed by Yang and collaborators ${ }^{30,31}$ ) and 
depends on a crucial insight of Levy ${ }^{35}$ regarding excitedstate derivative discontinuities. The latter connection employs a noncollinear version of the Casida equations, ${ }^{39}$ which we evaluate, again for the Lithium atom, within the singlepole approximation, in LSDA and for exact exchange.

The development of approximate density functionals and computational methodologies that permit the reliable calculation of spin gaps and related quantities, including their many-body $(x c)$ corrections, remains a challenge for the future.

\section{ACKNOWLEDGMENTS}

K.C. thanks the Physics Department of the University of Missouri-Columbia, where part of this work was done, for generous hospitality, and D. Vieira for preparing and discussing Fig. 1. K.C. is supported by Brazilian funding agencies FAPESP and CNPq. C.A.U. acknowledges support from NSF Grant No. DMR-0553485. C.A.U. would also like to thank the KITP Santa Barbara for its hospitality and partial support under NSF Grant No. PHY05-51164. G.V. acknowledges support from NSF Grant No. DMR-0705460.
${ }^{1}$ The expression "charge gap" is actually a misnomer, as what is added, removed or excited is a particle (with charge and spin) not just charge. Charge gap is the common expression, however, and we will use it interchangeably with the more correct "particle gap." In one-dimensional systems, on the other hand, it is important to distinguish charge and particle gaps, due to the possibility of spin-charge separation.

${ }^{2}$ R. M. Dreizler and E. K. U. Gross, Density Functional Theory (Springer, Berlin, 1990).

${ }^{3}$ R. G. Parr and W. Yang, Density-Functional Theory of Atoms and Molecules (Oxford University Press, Oxford, 1989).

${ }^{4}$ G. F. Giuliani and G. Vignale, Quantum Theory of the Electron Liquid (Cambridge University Press, Cambridge, England, 2005).

${ }^{5}$ J. P. Perdew, R. G. Parr, M. Levy, and J. L. Balduz, Phys. Rev. Lett. 49, 1691 (1982).

${ }^{6}$ J. P. Perdew and M. Levy, Phys. Rev. Lett. 51, 1884 (1983).

${ }^{7}$ L. J. Sham and M. Schlüter, Phys. Rev. Lett. 51, 1888 (1983).

${ }^{8}$ L. J. Sham and M. Schlüter, Phys. Rev. B 32, 3883 (1985).

${ }^{9}$ A. Seidl, A. Görling, P. Vogl, J. A. Majewski, and M. Levy, Phys. Rev. B 53, 3764 (1996).

${ }^{10}$ K. Capelle, M. Borgh, K. Karkkainen, and S. M. Reimann, Phys. Rev. Lett. 99, 010402 (2007).

${ }^{11}$ F. P. Rosselli, A. B. F. da Silva, and K. Capelle, arXiv:physics/ 0611180 (unpublished).

${ }^{12}$ J. Chen, J. B. Krieger, R. O. Esquivel, M. J. Stott, and G. J. Iafrate, Phys. Rev. A 54, 1910 (1996).

${ }^{13}$ J. B. Krieger, Y. Li, and G. J. Iafrate, Phys. Rev. A 45, 101 (1992).

${ }^{14}$ O. V. Gritsenko and E. J. Baerends, J. Chem. Phys. 120, 8364 (2004).

${ }^{15}$ S. Mannervik and H. Cederquist, Phys. Scr. 27, 175 (1983).

${ }^{16}$ C. F. Bunge and A. V. Bunge, Phys. Rev. A 17, 816 (1978); C. F. Bunge, J. Phys. B 14, 1 (1981); J.-J. Hsu, K. T. Chung, and K.-N. Huang, Phys. Rev. A 44, 5485 (1991).

${ }^{17}$ J. M. D. Coey and M. Venkatesan, J. Appl. Phys. 91, 8345 (2002).

${ }^{18}$ U. von Barth and L. Hedin, J. Phys. C 5, 1629 (1972).

${ }^{19}$ H. Eschrig and W. E. Pickett, Solid State Commun. 118, 123 (2001).

${ }^{20}$ K. Capelle and G. Vignale, Phys. Rev. Lett. 86, 5546 (2001).

${ }^{21}$ K. Capelle and G. Vignale, Phys. Rev. B 65, 113106 (2002).

${ }^{22}$ C. A. Ullrich, Phys. Rev. B 72, 073102 (2005).

${ }^{23}$ N. Argaman and G. Makov, Phys. Rev. B 66, 052413 (2002).
${ }^{24}$ N. I. Gidopoulos, Phys. Rev. B 75, 134408 (2007).

${ }^{25}$ W. Kohn, A. Savin, and C. A. Ullrich, Int. J. Quantum Chem. 100, 20 (2004).

${ }^{26}$ K. Capelle, C. A. Ullrich, and G. Vignale, Phys. Rev. A 76, 012508 (2007).

${ }^{27}$ T. Gál, P. W. Ayers, F. De Proft, and P. Geerlings, J. Chem. Phys. 131, 154114 (2009).

${ }^{28}$ J. P. Perdew and E. Sagvolden, Can. J. Chem. 87, 1268 (2009).

${ }^{29}$ G. K. L. Chan, J. Chem. Phys. 110, 4710 (1999).

${ }^{30}$ A. J. Cohen, P. Mori-Sánchez, and W. Yang, Science 321, 792 (2008).

${ }^{31}$ P. Mori-Sánchez, A. J. Cohen, and W. Yang, Phys. Rev. Lett. 102, 066403 (2009)

${ }^{32}$ A. K. Theophilou, J. Phys. C 12, 5419 (1979).

${ }^{33}$ E. K. U. Gross, L. N. Oliveira, and W. Kohn, Phys. Rev. A 37, 2805 (1988); 37, 2809 (1988); L. N. Oliveira, E. K. U. Gross, and W. Kohn, ibid. 37, 2821 (1988).

${ }^{34}$ A. Nagy, Phys. Rev. A 49, 3074 (1994); 42, 4388 (1990).

${ }^{35}$ M. Levy, Phys. Rev. A 52, R4313 (1995).

${ }^{36}$ Time-Dependent Density Functional Theory, Lecture Notes in Physics Vol. 706, edited by M. A. L. Marques, C. A. Ullrich, F. Nogueira, A. Rubio, K. Burke, and E. K. U. Gross (Springer, Berlin, 2006)

${ }^{37}$ P. Elliott, K. Burke, and F. Furche, in Recent Advances in Density Functional Methods, edited by K. B. Lipkowitz and T. R. Cundari (Wiley, Hoboken, NJ, 2009), Vol. 26, p. 91.

${ }^{38}$ M. E. Casida, in Recent Advances in Density Functional Methods, edited by D. E. Chong (World Scientific, Singapore, 1995), Vol. 1, p. 155.

${ }^{39}$ F. Wang and T. Ziegler, J. Chem. Phys. 121, 12191 (2004); 122, 074109 (2005); Int. J. Quantum Chem. 106, 2545 (2006).

${ }^{40}$ J. Sticht, K. H. Höck, and J. Kübler, J. Phys.: Condens. Matter 1, 8155 (1989).

${ }^{41}$ L. M. Sandratskii, Adv. Phys. 47, 91 (1998).

${ }^{42}$ O. Heinonen, J. M. Kinaret, and M. D. Johnson, Phys. Rev. B 59, 8073 (1999).

${ }^{43}$ C. A. Ullrich and M. E. Flatté, Phys. Rev. B 66, 205305 (2002); 68, 235310 (2003).

${ }^{44}$ M. Petersilka, U. J. Gossmann, and E. K. U. Gross, Phys. Rev. Lett. 76, 1212 (1996).

${ }^{45}$ H. Appel, E. K. U. Gross, and K. Burke, Phys. Rev. Lett. 90, 043005 (2003)

${ }^{46}$ Y.-H. Kim and A. Görling, Phys. Rev. Lett. 89, 096402 (2002).

${ }^{47}$ M. Hellgren and U. von Barth, Phys. Rev. B 78, 115107 (2008). 
${ }^{48}$ J. E. Sansonetti, W. C. Martin, and S. L. Young, Handbook of Basic Atomic Spectroscopic Data (National Institute of Standards and Technology, Gaithersburg, 2005).
${ }^{49}$ N. T. Maitra, F. Zhang, R. J. Cave, and K. Burke, J. Chem. Phys. 120, 5932 (2004).

${ }^{50}$ N. T. Maitra, J. Chem. Phys. 122, 234104 (2005). 